\title{
Overdose Education and Naloxone for Patients Prescribed Opioids in Primary Care: A Qualitative Study of Primary Care Staff
}

\author{
Ingrid A. Binswanger, MD, MPH ${ }^{1,2,3}$, Stephen Koester, $P h D^{4,5}$, Shane R. Mueller, MSW',2,5, \\ Edward M. Gardner, $M D^{3}$, Kristin Goddard, $M P H^{7}$, and Jason M. Glanz, $P h D^{1,6}$
}

\begin{abstract}
'Institute for Health Research, Kaiser Permanente Colorado, Denver, CO, USA; ${ }^{2}$ Division of General Internal Medicine, University of Colorado Denver School of Medicine, Aurora, CO, USA; ${ }^{3}$ Denver Health and Hospital Authority, Denver, CO, USA; ${ }^{4}$ Department of Anthropology, University of Colorado Denver, Denver, CO, USA; ${ }^{5}$ Department of Health and Behavioral Sciences, University of Colorado Denver, Denver, CO, USA;

${ }^{6}$ Department of Epidemiology, Colorado School of Public Health, Aurora, CO, USA.
\end{abstract}

BACKGROUND: The rate of fatal unintentional pharmaceutical opioid poisonings has increased substantially since the late 1990s. Naloxone is an effective opioid antidote that can be prescribed to patients for bystander use in the event of an overdose. Primary care clinics represent settings in which large populations of patients prescribed opioids could be reached for overdose education and naloxone prescription.

OBJECTIVE: Our aim was to investigate the knowledge, attitudes and beliefs about overdose education and naloxone prescription among clinical staff in primary care.

DESIGN: This was a qualitative study using focus groups to elucidate both clinic-level and provider-level barriers and facilitators.

SETTING: Ten primary care internal medicine, family medicine and infectious disease/HIV practices in three large Colorado health systems.

METHODS: A focus group guide was developed based on behavioral theory. Focus group transcripts were coded for manifest and latent meaning, and analyzed for themes using a recursive approach that included inductive and deductive analysis.

RESULTS: Themes emerged in four content areas related to overdose education and naloxone prescription: knowledge, barriers, benefits and facilitators. Clinical staff $(\mathrm{N}=$ 56) demonstrated substantial knowledge gaps about naloxone and its use in outpatient settings. They expressed uncertainty about who to prescribe naloxone to, and identified a range of logistical barriers to its use in practice. Staff also described fears about offending patients and concerns about increased risk behaviors in patients prescribed naloxone. When considering naloxone, some providers reflected critically and with discomfort on their own opioid prescribing. These barriers were balanced by beliefs that prescribing naloxone could prevent death and result in safer opioid use behaviors.

LIMITATIONS: Findings from these qualitative focus groups may not be generalizable to other settings.

CONCLUSION: In addition to evidence gaps, logistical and attitudinal barriers will need to be addressed to enhance uptake of overdose education and naloxone prescription for patients prescribed opioids for pain.

Received January 5, 2015

Revised February 24, 2015

Accepted April 30, 2015

Published online June 9, 2015
KEY WORDS: opioids; primary care; HIV; naloxone; overdose; qualitative research.

J Gen Intern Med 30(12): 1837-44

DOI: $10.1007 /$ s11606-015-3394-3

(C) Society of General Internal Medicine 2015

\section{INTRODUCTION}

Pharmaceutical opioid prescribing and fatal unintentional poisonings have increased substantially. ${ }^{1-4}$ Deaths from opioid poisonings may be prevented with naloxone, an effective, Food and Drug Administration approved opioid antidote with few contraindications to its use or serious adverse effects. ${ }^{5}$ Naloxone has traditionally been administered by first responders to reverse opioid-induced respiratory depression. Increasingly, community-based and public health programs have distributed naloxone for bystander administration, alongside education about the signs of an overdose, indications for use and administration directions. ${ }^{6}$ Program evaluations suggest that community-based overdose education and naloxone distribution is an effective strategy to prevent overdose fatalities. $^{7-12}$

Community-based programs have traditionally served people who use heroin and nonmedical opioids, but a significant proportion of overdose deaths are related to opioids prescribed for pain. ${ }^{13-17}$ In the Veteran's Administration and large managed care organizations, the overdose risk is particularly high in people prescribed more than 100 milligrams morphine equivalent daily doses. ${ }^{15,16}$ Primary care practices represent settings in which overdose education and naloxone prescribing could reach many patients prescribed opioids, but many scientific questions remain about widespread implementation of naloxone in primary care.

Although there are limited data on patient outcomes with primary-care - based naloxone prescription, new federal guidance exists on naloxone prescribing to patients prescribed opioids. ${ }^{18,19}$ To date, there is little evidence that primary care providers have engaged in widespread naloxone prescribing. Surveys in 2002-2003 demonstrated that few medical providers were knowledgeable about prescribing naloxone to prevent overdose, were willing to prescribe naloxone, or supported naloxone distribution. ${ }^{20-22}$ While these surveys were 
conducted early in the overdose epidemic and did not focus on patients prescribed opioids, their findings suggest that there may be considerable barriers to naloxone prescribing in primary care. To better understand these barriers, we conducted a qualitative study to assess clinical staff's knowledge, attitudes and beliefs about overdose education and naloxone prescribing.

\section{METHODS}

\section{Design Overview, Setting, and Participants}

Between August 2013 and October 2014, we conducted ten focus groups with clinical staff from a large public healthcare system (Denver Health Medical Center), a managed care organization (Kaiser Permanente Colorado), and an academic medical center (University of Colorado Hospital). We recruited participants from internal medicine, family medicine and HIV practices (as HIV infection is associated with an increased risk of overdose mortality). ${ }^{23-26}$ We emailed practice administrators, who identified convenient dates for attendance by available clinical staff. Focus groups were scheduled over lunch, provided as compensation. Clinic staff were given information about the study and invited to participate via email.

We conducted focus groups to understand issues related to naloxone prescribing at clinic (e.g., scheduling barriers, staff roles in patient care) and provider (e.g., specific attitudes) levels. Focus groups included diverse members of the health care team (e.g., physicians, nurses, pharmacists and administrators). The objective was to generate discussion and reveal individual and shared knowledge, attitudes and beliefs.

\section{Focus Group Content and Structure}

Our multidisciplinary team of researchers and clinicians developed a focus group guide (Appendix) informed by two theoretical frameworks: the Theory of Planned Behavior ${ }^{27}$ and the Health Belief Model. ${ }^{28}$ Informed consent was obtained, and participants completed a brief demographic survey. The primary facilitator of each focus group was a doctoral level anthropologist (S.K.) or a doctoral student with training in qualitative methods (S.M.). Three other authors (I.B., E.G., or K.G.) participated as co-facilitators in one or more of the focus groups.

Participants identified their clinic role at the beginning of the focus groups and facilitators elicited role-specific comments. Given the heterogeneity in clinical roles, some participants might have been uncomfortable discussing unfamiliar topics. To encourage open communication, our introduction emphasized that there were no right or wrong answers, that participants could ask questions, and that we were interested in their perspectives. Later, we explained what naloxone was and how it could be used.

\section{Data Analysis}

Focus groups were digitally recorded, professionally transcribed and entered into ATLAS.ti software. We employed an ethnographic, or recursive, strategy for data analysis. Recursive analysis is a cyclical, iterative process combining deductive and inductive approaches. ${ }^{29}$ A deductive or "top down" approach was used to link text to predefined codes and categories based on literature, prior knowledge, theoretical models and interview guide (e.g., time constraints are a barrier). An inductive or "bottom up" approach was used to identify new codes and categories that emerged from the data, ${ }^{29}$ including unanticipated information relevant to our research questions and theoretical models (e.g., concerns about patient risk behavior). Results describing attitudinal and contextual concerns of providers generally materialized from an inductive approach. Most other findings were derived from deductive analysis.

The anthropologist (S.K.), doctoral student (S.M.) and Master's level research assistant (K.G.) created an a priori template of codes informed by our theoretical models. ${ }^{30}$ Using this template as a guide, the three analysts independently coded two transcripts by assigning predefined codes to text and assigning new codes to emergent findings. This resulted in a revised code list that was applied to the remaining eight transcripts. In frequent meetings of the entire research team (including two physicians and an epidemiologist), the team discussed coding inconsistencies, refined the coding scheme, and ensured consensus. ${ }^{31}$ Codes were subsequently categorized into larger groupings, representing themes. Each transcript was summarized for salient themes and compared across focus groups. Themes that emerged from this process are reported here. ${ }^{29}$ We coded data for manifest content meaning (surface content, e.g., staff who stated they had limited knowledge of naloxone) and latent content meaning (underlying meaning, e.g., staff who demonstrated knowledge gaps). ${ }^{32}$ Analyzing data on both levels was particularly important for understanding potential barriers to naloxone prescription.

This study was approved by the Colorado Multiple Institutional Review Board and the Kaiser Permanente Colorado Institutional Review Board. We received a Federal Certificate of Confidentiality.

\section{RESULTS}

We enrolled 56 participants (Table 1). All focus groups included at least one prescriber (physician, nurse practitioner, or physician's assistant). Three ( $8 \%$ ) of the 37 participants with prescribing authority had prescribed naloxone. We did not observe substantial differences in themes based on clinic roles, nor areas of disagreement in focus groups that could be linked to roles. For ease of reporting, themes are organized into the following four content areas related to overdose education and naloxone prescription: 1) knowledge, 2) barriers, 3) benefits and 4) facilitators. 
Table 1. Participant Characteristics $(\mathrm{N}=56)$

\begin{tabular}{ll}
\hline \hline Age in years, mean (standard deviation [SD]) & $40.8(9.7)$ \\
Female, no. (\%) & $33(58.9)$ \\
Race/ethnicity, no. (\%) & \\
White, non-Hispanic & $47(83.9)$ \\
African American, non-Hispanic & $2(3.6)$ \\
Hispanic & $3(5.4)$ \\
Asian, non-Hispanic & $4(7.1)$ \\
Professional role, no. (\%) & $31(55.4)$ \\
Physician & $6(10.7)$ \\
Nurse & $7(12.5)$ \\
Pharmacist & $4(7.1)$ \\
Nurse Practitioner & $3(5.4)$ \\
Clinic Administrator & $2(3.6)$ \\
Counselor & $2(3.6)$ \\
Physician Assistant & $1(1.8)$ \\
Medical Assistant & $12.0(8.9)$ \\
Years since terminal degree, mean (SD) & $15(32.6)$ \\
Prior receipt of education about naloxone for take & \\
home use, no. (\%) & $3(8.1)$ \\
Among prescribers (n=37), ever prescribed naloxone, & \\
no. (\%) &
\end{tabular}

\section{Knowledge}

Limited Knowledge About Naloxone for Bystander Use. Clinical staff had limited awareness and clinical knowledge about outpatient naloxone prescribing. Some expressed confusion between naloxone for overdose and addiction medications (e.g., naltrexone). Generally, providers last used naloxone during medical training in emergency departments. Among providers in the public healthcare system, there was greater familiarity with naloxone because it was available on the outpatient formulary. Clinical staff expressed concerns about abuse and diversion of naloxone, and fears of serious adverse events including cardiac arrest and seizures:

"I probably just don't have quite as much knowledge about the outpatient safety of it to feel comfortable prescribing it right now." (Physician)

As a result, few had prescribed naloxone.

Level of Knowledge About Overdose Events Among Clinic Patients Influenced Risk Perception. In many practices, participants were uncertain about whether patients in their practice had experienced overdose from prescribed opioids:

"I don't know if they are overdosing. I guess if they do, they're not telling us about it probably." (Physician)

Communication gaps between clinical departments contributed to poor knowledge about overdoses. Yet, staff with greater knowledge about events among practice patients, particularly recent events, expressed greater risk perception.

"I think the risk of overdosing is huge. How often our patients overdose, it's hard to know... since our ER hasn't necessarily talked to our clinic, and do they overdose, but not go receive care? I mean, they just sort of happen to get lucky and survive the overdose. But I would say that within the last couple of months, I know of three overdoses that have resulted in going to our ER." (Physician)
In a practice that monitors its patient mortality rate in an ongoing quality improvement project, staff had high risk perception:

"We are cognizant of the fact that overdoses, you know, are at least a possibility and it's always in the back of our minds." (Pharmacist)

Participants Identified Different Groups of Patients as Potentially at Risk for Overdose. Across the focus groups, participants identified at least nine risk groups for overdose, including patients (1) prescribed high-dose opioids, long-acting opioids, or benzodiazepines; (2) with a history of or predisposition to substance use disorders, or who also use alcohol or marijuana; and (3) with co-occurring mental health problems. One primary care provider explained:

"So, the things that would raise somebody's risk level would be first what doses are they on... if someone has a history of abnormal urine/drug screens or has a history of either abuse, drug abuse, or a lot of psychiatric stuff that the doctor is concerned about, they can raise them from a low to a moderate to a high risk level." (Physician)

Participants identified additional risk groups, such as patients with (4) challenging or unstable social circumstances; (5) no access to ancillary pain services (e.g., physical therapy, acupuncture, psychotherapy); (6) behavioral characteristics, such as poor coping skills or impulsivity; (7) unrealistic expectations about the efficacy of opioids to control pain; and (8) inadequate attention to or understanding of safe use:

"Some of our patients who sort of medicate every symptom are a big issue... The other people who are at risk for overdose is that cavalier attitude towards opiates and lack of concern for safety." (Physician)

Finally, patients with (9) uncontrolled pain were considered at risk. In uncontrolled pain, inadequate patient education and poor health literacy were thought to contribute to risk:

"I think people [are at risk] who...either because we have not explained it well, or they're unreceptive or unclear about medication administration. I think certainly they can end up being in a compromised safety situation because of trying to control pain and just not feeling better so popping another and popping another and popping another." (Nurse)

Whereas staff generally emphasized individual risk characteristics, others included social or family context. For instance, one provider thought patients with family members who 
could access the patient's medications should be prescribed naloxone:

"I had a patient whose daughter accidentally overdosed on her meds, so, I'm wondering, shouldn't we be offering it [naloxone] more broadly? ...Do we have this discussion with everybody and then offer to write the prescription for those who are accepting of it?" (Physician)

Some participants suggested universal risk among patients prescribed opioids: "Yeah, I'd say every patient that is taking opioids" is at risk for opioid overdose. Another participant suggested universal prescribing to patients on opioids.

"I mean logistically it's hard to reach out to every patient, but if the goal is to save lives, you have to bring it up to everybody." (Pharmacist)

\section{Barriers}

Logistical and Systems Barriers. Logistical and systems barriers to naloxone prescribing included time, privacy, how to remember to prescribe naloxone, factors that might prevent effective naloxone use, and appropriate clinical follow-up after an event (Table 2).

Attitudinal and Contextual Concerns. Providers expressed discomfort with prescribing naloxone, in contrast to other medications prescribed in outpatient settings for emergency administration:

"It seems kind of intuitive, like glucagon for insulin. But it just feels a little uncomfortable where glucagon just doesn't." (Physician)

We identified three themes related to attitudes and the opioid prescribing context that led to this discomfort: (1) fears of offending patients, (2) concerns about patients engaging in greater risk behavior, and (3) difficulty reconciling the implications of naloxone with current opioid prescribing practice.

Clinical staff were concerned that discussing overdose and prescribing naloxone would negatively impact patients' opinions of their providers (theme 1 ):

"I feel that patients would be almost offended, like, oh, you're singling me out and I'm cherry picked to do this." (Pharmacist)

Providers were concerned about poor patient satisfaction ratings:

"The barrier is going to be the overdose discussion without sacrificing customer satisfaction and customer service." (Counselor)
Table 2. Logistical and Systems Barriers to Naloxone Prescription in Primary Care

\begin{tabular}{l}
\hline Barrier \\
\hline $\begin{array}{l}\text { Integrating naloxone into busy } \\
\text { clinical schedules }\end{array}$ \\
$\begin{array}{l}\text { Difficulty remembering to discuss } \\
\text { naloxone and competing needs }\end{array}$
\end{tabular}

Training bystanders and uncertainty about whether bystanders are available

Difficulty assembling the device for intranasal administration

Lack of confidentiality

Uncertainty about billing for the drug, device and training

Patient costs

Limited availability of naloxone

Uncertainty about bystanders having naloxone available, identifying an overdose, using naloxone properly, delaying calling 911 and doing rescue breathing

Illustrative Quotation

"I don't think I'd have the time, no... These patients are [the] most time consuming patients." (Physician)

"It's so much to talk about, it doesn't really lend itself to be just incorporated into usual care that well. Most of our patients have, you know, multiple comorbidities that they're coming in for and frequently coming in in crisis about this, that and the other." (Physician)

"The idea that you teach the person that's taking the narcotics and relying on them to teach the other person is kind of a limiting factor in how successful it is." (Pharmacist)

Respondent 1: “...might then be hard to have them bring in the family member, you know, to go over it."

Respondent 2: "Or, the homeless population who don't have anybody." (Physician)

"We assemble it in front of them and say... this is how you should assemble this and, you know, put it in my nose and inject it. So that's the education part we give, but is that adequate? No, it's not because you know they're teaching that person that will actually administering this drug and that's one of the shortcomings of this." (Pharmacist)

"[In the pharmacy] we do have a little divider that gives you a little privacy, but, you know, everybody's definition of how much privacy is enough, but we do teach them at the counter." (Pharmacist)

"We talked about making a video or maybe doing groups run by the nurse or the pharmacist, but then the privacy issues came up." (Physician)

"Billing for the training cause that's going to be time....it has to be somehow reimbursable for the time it takes." (Physician)

"Insurance coverage."

(Administrator)

"Pharmacy availability."

(Administrator)

Respondent 1 (Nurse Practitioner): "Having it with them. I mean you're assuming that an overdose is going to happen at home, which may not necessarily... it may be at their partner's house, they may be, you know, God only knows."

Respondent 2 (Nurse Practitioner): "Partying..."

Respondent 1: "At a bar, who knows? So access."

Staff emphasized that messaging to patients would need to counter the stigma associated with being prescribed naloxone: 
"We have to always tell them, this is a new program and it's for everybody. It's not just for you. We're not saying you're a druggie or anything like that." (Pharmacist)

Overall, provider fears about being perceived negatively by patients reduced their willingness to discuss overdose and prescribe naloxone.

Another important barrier to naloxone prescription was giving patients a "false sense of security" which could lead to riskier use of opioids and more adverse events (theme 2). One physician said:

"Will it [naloxone] lead to more overdoses? Is there a chance that they'll potentially inject themselves [with naloxone] so they can take more [opioids] and then it [the naloxone] runs out. You know, I wonder... Patients do stupid things all the time to get high. Will this be one of them?" (Physician)

Another provider said:

"One of the concerns I would have was does that give them license to kind of just party away and expect a friend to save their life and they just go to the edge? Are they going to take more risk?" (Physician)

As a result, providers expressed more comfort prescribing naloxone to patients who were not necessarily those who could benefit the most from it:

"I think the people that I would give the [naloxone] to would be people that are concerned, responsible and they're probably not the ones that are going to overdose." (Physician)

Other clinical staff rejected the concern about naloxone prompting riskier behaviors in light of its potential public health benefit:

"There were 14,000 deaths on opiate overdoses last year, so obviously people are dying... there's the cost of it [naloxone] is pretty low and the potential benefit is pretty high... We can't control what our patients do and if they happen to unfortunately overdose, I think it would probably be a good thing for someone around them to have [naloxone] 'cause the alternative is, they don't have it and they go into respiratory depression and die... It seems like practically it's a bit of a no brainer." (Physician)

Finally, providers struggled to reconcile the implications of prescribing naloxone with their profession's opioid prescribing practices (theme 3 ). They perceived a conflict between prescribing opioids and an opioid antidote. For instance, two providers had this exchange:

Respondent 1: "Like, if you're worried about them overdosing on it, don't prescribe it [opioids] to them." (Physician)
Respondent 2: "Exactly. That's the thing. It feels like if I'm looking at you in the face and I'm going to send you home with a script [for opioids] that..." (Physician)

Respondent 1: "Could kill you.” (Physician)

Identifying patients at risk also made providers reconsider prescribing opioids to those same patients.

"It [naloxone] seems like it may have merit. On some level, it also makes me feel like it's sort of putting our head in the sand just a little bit, you know. If you feel like, my God, this patient is going to kill themselves, maybe the solution is to not have them on opioids." (Physician)

Providers expressed concern that they would be treating the "symptom rather than the problem" of opioid over-prescribing and inadequate access to ancillary pain services.

"I think we can have a bigger impact from a population standpoint if we actually do think more about when we're prescribing these meds." (Physician)

Consequently, providers wondered if opioids should be reduced among "at risk" patients. Further, one provider was concerned about medico-legal risk if a patient was identified as "at risk", prescribed naloxone, and continued on opioids, particularly if he or she experienced a subsequent overdose.

\section{Benefits}

Based on their experience with naloxone in emergency settings, staff commonly expressed the belief that naloxone could prevent overdose deaths, through its direct therapeutic effect.

"It [naloxone] will make you feel terrible. But it'll save your life." (Physician)

Additionally, clinical staff suggested indirect benefits of discussing and prescribing naloxone, by influencing patients' understanding of the risks of opioids, opioid use behavior and cultural norms related to safety. One primary care provider illustrated both direct and indirect benefits:

"I was sort of hoping that if we implement a good program where even at initiation [of opioids], we talk about overdose prevention and naloxone, that it will bring, you know, the safety concerns to the forefront, and then it might actually help people understand that these are 
potentially lethal medications, and I feel like that might be one of the things that might be most beneficial from it... just re-setting of, like, the culture around these medications [opioids] as much as, you know, potentially saving someone's life from overdose." (Physician)

Another provider endorsed sending a message to patients as an indirect benefit of discussing naloxone:

"Just that conversation... might be just enough to scare them just a little." (Nurse)

Given these potential benefits, participants were receptive to naloxone use despite the barriers.

\section{Facilitators}

Participants identified three potential facilitators to naloxone prescribing. Providers desired guidelines that could be applied in a standard fashion across patients, providers and systems.

"So I would want there to be guidelines in place... institutionally sanctioned as to how to risk stratify patients and what the appropriate prescribing guidelines would be." (Nurse Practitioner)

Further, staff suggested reducing the stigma of naloxone prescribing by including household members as potential recipients:

"Another thing that might be a little bit less destigmatizing for patients is also saying, it's not just for you if you accidentally overdose, but if someone you know or someone in your household takes your medications, you know, then you, you know, have the means." (Physician)

Additionally, providers requested improved communication from emergency departments about overdoses among their patients, and guidance on opioid management after these events.

"If they get in that situation that they would need that medication [naloxone], what's the follow-up after that? You know, generally there's an emergency room. You have follow-up and you have that conversation, otherwise they're just at home and nothing changes." (Nurse Practitioner)

\section{DISCUSSION}

Overall, primary care staff suggested several potential benefits of overdose education and naloxone prescription among patients prescribed opioids. These included enhancing patient understanding of the risks of opioids, promoting safer use behaviors, and preventing mortality. While providers had few concerns about naloxone's efficacy, our study identified key knowledge, attitudinal and contextual barriers that limited enthusiasm for naloxone.

Notably, an important barrier that emerged was a lack of consensus about who should be prescribed naloxone. Clinical staff identified a wide and complex range of risk factors, demonstrating a need for additional tools to help identify patients who could benefit. However, focusing on individual risk factors may inhibit naloxone prescription because it targets patient behavior, in contrast to treating respiratory depression as a known adverse event of opioid medications. ${ }^{33}$ One potential solution is developing standardized naloxone patient selection protocols, which could lessen provider fears about offending patients and facing medico-legal consequences. Another potential solution raised was universal prescribing, in which naloxone would be indicated for all patients prescribed chronic opioids.

In theory, naloxone prescribing could increase risk behaviors in patients prescribed opioids. This effect, commonly known as risk compensation, ${ }^{34}$ has been raised in response to preventive interventions, such as vaccination against human papilloma virus and pre-exposure prophylaxis for HIV prevention. ${ }^{35-37}$ However, prescribing naloxone could also represent an opportunity to increase patients' risk perceptions of opioids. Further research is needed to determine whether practice-based naloxone prescribing increases, reduces or has no effect on opioid-related risk behavior.

Several providers in our study uncomfortably deliberated on opioid prescribing in light of the potential need to prescribe an antidote. We interpreted this effect as a form of cognitive dissonance, ${ }^{38}$ which may lead providers to become more judicious about how they prescribe opioids. This effect, however, may also have a negative impact on patients if providers reduce or stop prescribing to those who benefit from opioids or initiate rapid tapers that lead to uncomfortable withdrawal symptoms. Our findings suggest a delicate balance between the potential benefits and drawbacks of naloxone in primary care, which should be empirically assessed in future research.

We sought to identify the breadth of issues from the perspectives of the clinical stakeholders rather than quantify or test a hypothesis. Including three health systems helped ensure that perspectives from diverse practice settings were assessed. Our study has limitations, however. Our findings should not be assumed to represent the opinions of the entire clinical staff, as some members may have felt uncomfortable speaking openly in focus groups. Furthermore, this study was conducted in a state that passed legislation designed to widen access to naloxone. $^{39}$ To the extent that participants were aware of this legislation, it may have positively affected attitudes towards naloxone. 
Naloxone is an efficacious medication that has the potential to prevent deaths among patients prescribed opioids in primary care settings. However, our study identified important knowledge, attitude and contextual barriers that may hinder naloxone prescription and use in these settings. Although the Substance Abuse and Mental Health Services Administration has issued a toolkit with information on naloxone for providers that addresses knowledge gaps, ${ }^{18}$ this resource does not fully address attitudinal and contextual barriers. Further research is needed to address the questions raised by providers, such as whether prescribing naloxone will negatively impact patient satisfaction, whether patients engage in risk compensation, and whether there are safety implications of increased prescribing. In addition, the effectiveness of naloxone prescription to patients prescribed opioids in primary care should be evaluated. Finally, the role of naloxone prescription should be evaluated in the context of other potential modalities to promote opioid safety and reduce the risk of overdose.

Acknowledgements: Funding Source: This work was supported by the National Institute on Drug Abuse, which was not involved in the design, conduct, or reporting of the study, or in the decision to submit the manuscript for publication.

Grant Support: Research reported in this publication was supported by the National Institute on Drug Abuse of the National Institutes of Health under Award Number R34DA035952. The content is solely the responsibility of the authors and does not necessarily represent the official views of the National Institutes of Health.

Reproducible Research Statement: Study protocol: available from the corresponding author. Analytic code and data set: not available.

Author Contributions: Conception and design: I.A. Binswanger and J.M. Glanz.

Analysis and interpretation of the data: S. Koester, S. Mueller, K. Goddard, I.A. Binswanger J.M. Glanz and E.M. Gardner

Drafting of the article: I.A. Binswanger and J.M. Glanz

Critical revision of the article for important intellectual content: $S$. Koester, S. Mueller, K. Goddard, and E.M. Gardner

Final approval of the article: S. Koester, S. Mueller, K. Goddard, and E. Gardner

Administrative, technical, or logistic support: S. Mueller and K. Goddard.

Collection and assembly of data: S. Koester, S. Mueller, K. Goddard, and I.A. Binswanger

Potential Conflicts of Interest: The authors declare that they do not have a conflict of interest.

Corresponding Author: Ingrid A. Binswanger, MD, MPH; Institute for Health Research, Kaiser Permanente Colorado, P.O Box 378066, Denver, CO 80237-8066, USA (e-mail: Ingrid.A.B.inswanger@kp.org).

\section{REFERENCES}

1. Paulozzi LJ, Weisler RH, Patkar AA. A national epidemic of unintentional prescription opioid overdose deaths: how physicians can help control it. J Clin Psychiatry. 2011;72(5):589-92. doi:10.4088/JCP.10com06560.

2. Centers for Disease Control and Prevention. Vital signs: overdoses of prescription opioid pain relievers - United States, 1999-2008. MMWR. 2011;60:1487-92.
3. Paulozzi LJ. Drug-induced deaths-United States, 2003-2007. MMWR Surveill Summ. 2011;60(Suppl):60-1.

4. Calcaterra S, Glanz J, Binswanger IA. National trends in pharmaceutical opioid related overdose deaths compared to other substance related overdose deaths: 1999-2009. Drug Alcohol Depend. 2013;131(3):263-70. doi:10.1016/j.drugalcdep.2012. 11.018 .

5. Boyer EW. Management of opioid analgesic overdose. N Engl J Med. 2012;367(2): 146-55. doi:10.1056/NEJMra1202561.

6. Wheeler E, Davidson PJ, Jones S, Irwin KS. Community-based opioid overdose prevention programs providing naloxone - United States, 2010. MMWR. 2012;61:101-5.

7. Walley AY, Xuan Z, Hackman HH, Guinn E, Doe-Simkins M, SorensenAlawad A, et al. Opioid overdose rates and implementation of overdose education and nasal naloxone distribution in Massachusetts: interrupted time series analysis. BMJ. 2013;346:f174. doi:10. $1136 /$ bmj.f174.

8. McAuley A, Best D, Taylor A, Hunter C, Robertson R. From evidence to policy: The Scottish national naloxone programme. Drugs: Educ Prev Polic. 2012;19(4):309-19. doi:10.3109/09687637.2012.682232.

9. Bennett T, Holloway $\mathbf{K}$. The impact of take-home naloxone distribution and training on opiate overdose knowledge and response: An evaluation of the THN Project in Wales. Drugs: Educ Prev Polic. 2012;19(4):320-8. doi:10.3109/09687637.2012.658104.

10. Doe-Simkins M, Walley AY, Epstein A, Moyer P. Saved by the nose: bystander-administered intranasal naloxone hydrochloride for opioid overdose. Am J Public Health. 2009;99(5):788-91. doi:10.2105/AJPH. 2008.146647.

11. Albert S, Brason FW 2nd, Sanford CK, Dasgupta N, Graham J, Lovette B. Project Lazarus: community-based overdose prevention in rural North Carolina. Pain Med. 2011;12(Suppl 2):S77-85. doi:10.1111/j.1526-4637. 2011.01128.x.

12. Enteen L, Bauer J, McLean R, Wheeler E, Huriaux E, Kral AH, et al. Overdose prevention and naloxone prescription for opioid users in San Francisco. J Urban Health. 2010;87(6):931-41. doi:10.1007/s11524-0109495-8.

13. Hall AJ, Logan JE, Toblin RL, Kaplan JA, Kraner JC, Bixler D, et al. Patterns of abuse among unintentional pharmaceutical overdose fatalities. JAMA. 2008;300(22):2613-20. doi:10.1001/ jama.2008.802.

14. Baumblatt J, Wiedeman C, Dunn JR, Schaffner W, Paulozzi LJ, Jones TF. High-risk use by patients prescribed opioids for pain and its role in overdose deaths. JAMA Internal Medicine. 2014. doi:10.1001/ jamainternmed.2013.12711.

15. Dunn KM, Saunders KW, Rutter CM, Banta-Green CJ, Merrill JO, Sullivan MD, et al. Opioid Prescriptions for Chronic Pain and Overdose A Cohort Study. Ann. Intern. Med. 2010;152:85-92.

16. Bohnert AS, Valenstein M, Bair MJ, Ganoczy D, McCarthy JF, Ilgen MA, et al. Association between opioid prescribing patterns and opioid overdose-related deaths. JAMA. 2011;305(13):1315-21. doi:10.1001/ jama.2011.370.

17. Braden JB, Russo J, Fan M-Y, Edlund MJ, Martin BC, DeVries A, et al. Emergency Department Visits Among Recipients of Chronic Opioid Therapy. Arch Intern Med. 2010;170(16):1425-32. doi:10.1001/ archinternmed.2010.273.

18. Substance Abuse and Mental Health Services Administration. SAMHSA Opioid Overdose Prevention Toolkit. HHS Publication No. (SMA) 13-4742. Rockville, MD 2013.

19. Volkow N. Naloxone-A Potential Lifesaver. National Institute on Drug Abuse. Available at http://www.drugabuse.gov/about-nida/noras-blog/ 2014/02/naloxone-potential-lifesaver. Accessed February 9, 2014.

20. Beletsky L, Ruthazer R, Macalino GE, Rich JD, Tan L, Burris S. Physicians' knowledge of and willingness to prescribe naloxone to reverse accidental opiate overdose: challenges and opportunities. J Urban Health. 2007;84(1):126-36. doi:10.1007/s11524-006-9120-z.

21. Coffin PO, Fuller C, Vadnai L, Blaney S, Galea S, Vlahov D. Preliminary evidence of health care provider support for naloxone prescription as overdose fatality prevention strategy in New York City. J Urban Health. 2003;80(2):288-90.

22. Tobin KE, Gaasch WR, Clarke C, Mackenzie E, Latkin CA. Attitudes of Emergency Medical Service providers towards naloxone distribution programs. J Urban Health. 2005;82(2):296-302. doi:10.1093/jurban/ jti052.

23. Silverberg MJ, Ray GT, Saunders K, Rutter CM, Campbell CI, Merrill JO, et al. Prescription long-term opioid use in HIV-infected patients. Clin J Pain. 2012;28(1):39-46. doi:10.1097/AJP.0b013e3182201a0f. 
24. Wang C, Vlahov D, Galai N, Cole SR, Bareta J, Pollini R, et al. The effect of HIV infection on overdose mortality. AIDS. 2005; 19(9):935-42.

25. Green TC, McGowan SK, Yokell MA, Pouget ER, Rich JD. HIV infection and risk of overdose: a systematic review and meta-analysis. AIDS. 2012;26(4):403-17. doi:10.1097/QAD.0b013e32834f19b6.

26. French AL, Gawel SH, Hershow R, Benning LM, Hessol NA, Levine AM, et al. Trends in Mortality and Causes of Death Among Women With HIV in the United States: A 10-Year Study. JAIDS. 2009;51(4):399-406.

27. Montaño DE, Kasprzykl D. Theory of Reasoned Action, Theory of Planned Behavior, and the Integrated Behavioral Model, Chapter 4. In: Glanz K, Rimer BK, Lewis FM, eds. Health behavior and health education: theory, research, and practice. 4th ed. San Francisco: Jossey-Bass; 2008.

28. Champion VL, Skinner CS. The Health Belief Model, Chapter 3. In: Glanz K, Rimer BK, Lewis FM, eds. Health behavior and health education: theory, research, and practice. 4th ed. San Francisco: Jossey-Bass; 2008.

29. LeCompte MD, Schensul JJ. Analysis and interpretation of ethnographic data: A mixed methods approach. Vol. 5. 2nd ed. Rowman Altamira, 2012.

30. Fereday J, Muir-Cochrane E. Demonstrating rigor using thematic analysis: A hybrid approach of inductive and deductive coding and theme development. Int J Qual Methods. 2006;5(1):1-11.

31. Barbour RS. Checklists for improving rigour in qualitative research: a case of the tail wagging the dog? BMJ. 2001;322(7294):1115-7.

32. Cho JY, Lee E-H. Reducing confusion about grounded theory and qualitative content analysis: similarities and differences. Qual Rep. 2014; 19(32): 1-20.

33. Dowell D, Kunins HV, Farley TA. Opioid analgesics-risky drugs, not risky patients. JAMA. 2013;309(21):2219-20. doi:10.1001/jama. 2013.5794.

34. Hogben M, Liddon N. Disinhibition and risk compensation: scope, definitions, and perspective. Sex Transm Dis. 2008;35(12):1009-10. doi:10.1097/OLQ.0b013e31818eb752.
35. Marcus JL, Glidden DV, Mayer KH, Liu AY, Buchbinder SP, Amico KR, et al. No evidence of sexual risk compensation in the iPrEx trial of daily oral HIV preexposure prophylaxis. PloS one. 2013;8(12), e81997. doi:10.1371/ journal.pone.0081997.

36. Westercamp N, Agot K, Jaoko W, Bailey R. Risk Compensation Following Male Circumcision: Results from a Two-Year Prospective Cohort Study of Recently Circumcised and Uncircumcised Men in Nyanza Province Kenya. AIDS Behav. 2014;18(9):1764-75. doi:10.1007/s10461-014-0846-4.

37. Liddon NC, Leichliter JS, Markowitz LE. Human Papillomavirus Vaccine and Sexual Behavior Among Adolescent and Young Women. Am. J. Prev. Med. 2012;42(1):44-52. doi:10.1016/j.amepre.2011.09.024.

38. Harmon-Jones, EE, Mills J. Cognitive Dissonance: Progress on a Pivotal Theory in Social Psychology. Washington, DC: American Psychological Association. 1999.

39. Colo. Rev. Stat. § 12-36-117(1.7) (2013).

\section{APPENDIX}

Table 3. Focus Group Content Areas and Sample Questions

\begin{tabular}{ll}
\hline \hline Content area & Representative questions \\
\hline $\begin{array}{l}\text { Knowledge } \\
\text { Current practice }\end{array}$ & $\begin{array}{l}\text { What do you know about naloxone? } \\
\text { In your practice, how do you currently address drug } \\
\text { overdose? Why? }\end{array}$ \\
Severity & $\begin{array}{l}\text { How serious a problem is opioid overdose in your } \\
\text { patients? }\end{array}$ \\
Susceptibility & $\begin{array}{l}\text { Who do you think is at risk of overdose? } \\
\text { Benefits }\end{array}$ \\
$\begin{array}{l}\text { What benefits and risks do you see in prescribing } \\
\text { naloxone to your patients? }\end{array}$ \\
Barriers & $\begin{array}{l}\text { Have there been any barriers to counseling patients } \\
\text { in your practice about overdose or prescribing them } \\
\text { naloxone? }\end{array}$ \\
Implementation & $\begin{array}{l}\text { What kind of delivery model would work well with } \\
\text { overdose prevention? }\end{array}$ \\
\hline
\end{tabular}

